\title{
Genomic Signatures of Pregnancy-Associated Breast Cancer Epithelia and Stroma and their Regulation by Estrogens and Progesterone
}

\author{
Djuana M. E. Harvell • Jihye Kim • Jenean O'Brien • \\ Aik-Choon Tan • Virginia F. Borges - Pepper Schedin • \\ Britta M. Jacobsen • Kathryn B. Horwitz
}

Received: 12 July 2012 / Accepted: 6 February 2013 /Published online: 12 March 2013

(C) Springer Science+Business Media New York 2013

\begin{abstract}
Pregnancy-associated breast cancers (PABC) generally present at advanced stages and have a poor prognosis. The reasons are unclear but we hypothesized that the continuous high levels of estrogens and progesterone were involved. We have now carried out a detailed analysis of PABC compared to tumors of age-matched nonpregnant (non-PABC) women. Malignant epithelia and tumor-associated stroma of PABC and non-PABC were isolated by laser capture microdissection and gene expression profiled. Additionally, normal breast epithelia and stroma adjacent to the two tumor types were analyzed. Lastly, subsets of previously identified E- and P-regulated genes were defined in all tissues. We find that PABC signatures cluster with established breast cancer subtypes. Major hormone-regulated genes whose expression correlated with epithelia of PABC dealt with regulation of cell proliferation, metabolism, and tumor aggressiveness,
\end{abstract}

\footnotetext{
Electronic supplementary material The online version of this article (doi:10.1007/s12672-013-0136-z) contains supplementary material, which is available to authorized users.
}

D. M. E. Harvell $(\varangle) \cdot$ B. M. Jacobsen $\cdot$ K. B. Horwitz Divisions of Endocrinology, University of Colorado Anschutz Medical Campus, Mail Stop 8106, 12801 E. 17th Avenue, Aurora, CO 80045, USA

e-mail: djuana.harvell@ucdenver.edu

B. M. Jacobsen

e-mail: britta.jacobsen@ucdenver.edu

K. B. Horwitz

e-mail: kate.horwitz@ucdenver.edu

J. Kim · J. O’Brien • A.-C. Tan • V. F. Borges · P. Schedin

Medical Oncology, Department of Medicine, University of

Colorado Anschutz Medical Campus, Mail Stop 8106, 12801 E.

17 th Avenue,

Aurora, CO 80045, USA including genes used to predict tumor recurrence. Compared to normal epithelia, a significant number of genes associated with cell cycle processes were enriched in PABC, many of which are hormone regulated. Thus, compared to normal epithelia, many of the genes that were differentially expressed in epithelia of PABC were distinct from those differentially expressed in non-PABC. With regard to the tumor microenvironment, immune-related genes were enriched in tumorassociated stroma of PABC. Compared to normal stroma, PABC-associated stroma overexpressed immune response genes, while genes involved in angiogenesis and extracellular matrix deposition were more commonly downregulated. This suggests that the heightened aggressiveness of PABC may involve a predisposition to metastasis through extracellular matrix degradation, plus angiogenesis independence. Moreover, genes encoding cell proliferative factors, signaling,

J. Kim

e-mail: jihye.kim@ucdenver.edu

J. O’Brien

e-mail: jenean.obrien@ucdenver.edu

A.-C. Tan

e-mail: aikchoon.tan@ucdenver.edu

V. F. Borges

e-mail: virginia.borges@ucdenver.edu

P. Schedin

e-mail: pepper.schedin@ucdenver.edu

K. B. Horwitz

Department of Pathology, University of Colorado Anschutz Medical Campus, Mail Stop 8106, 12801 E. 17th Avenue,

Aurora, CO 80045, USA 
immunomodulators and cell death, were hormone regulated in stroma. In sum, these analyses demonstrate complex patterns of enrichment and hormonal regulation of genes in PABC and suggest that it may have a distinct biological nature.

$\begin{array}{ll}\text { Abbreviations } \\ \text { PABC } & \text { Pregnancy associated breast cancer } \\ \text { Non-PABC } & \text { Nonpregnancy associated breast cancer } \\ \text { E } & \text { Estrogen(s) } \\ \text { P } & \text { Progesterone(s) } \\ \text { ER } & \text { Estrogen receptor } \\ \text { PR } & \text { Progesterone receptor } \\ \text { ECM } & \text { Extracellular matrix } \\ \text { LCM } & \text { Laser capture microdissection } \\ \text { RMA } & \text { Robust multi-array average } \\ \text { SNR } & \text { Signal-to-noise ratio } \\ \text { GSEA } & \text { Gene set-enrichment analysis } \\ \text { KEGG } & \text { Kyoto Encyclopedia of Genes and Genomes } \\ \text { GO } & \text { Gene ontology }\end{array}$

\section{Introduction}

Pregnancy associated breast cancers (PABC) variably include cases diagnosed during gestation, lactation, within 1 year of delivery, or up to $5-10$ years postpartum $[1,2]$. In the European registry, the average age of patients with PABC is 33 years (range, 23-47 years) and the median gestation age at diagnosis is 21 weeks [2]. Among all breast cancer patients, $0.2-3.8 \%$ have a pregnancy-related disease, comprising 1:10,000-1:3,000 pregnancies and about 3,500 cases in the USA each year [3]. However, among premenopausal women - the only population at risk for this overlap - the numbers are much higher, with 10-25\% of breast cancers estimated to be pregnancy-associated when defined as pregnant or within 1 year [3]. The postpartum subset of PABC, those diagnosed after, but within 5 years of a completed pregnancy, represents $40-50 \%$ of all breast cancer cases under age 45 [1]. Some studies suggest that older maternal age at first pregnancy and a family history or carrying a BRCA2 mutation represent a specific high-risk group for PABC [1].

The most common (75-90 \%) histologic type of PABC is invasive ductal carcinoma, with the majority of women diagnosed during or immediately following a completed pregnancy, often with advanced stage disease [2]. PABC is also characterized by higher (56-89 \%) rates of lymph node involvement compared to nonpregnant (non-PABC), agematched women (38-55\%) [2]. Estrogen (ER) and progesterone receptors (PR) are often negative ( $\sim 70 \%$ of tumors) in PABC [2]. However whether ER negativity is increased above the rate expected in age-matched non-PABC controls is unclear, and the high negativity rate may reflect cases diagnosed strictly during pregnancy [4]. These tumors may be falsely classified as ER and PR negative due to downregulation of the receptors in the face of high circulating estrogen (E) and progesterone (P) levels. Liganddependent ER and PR downregulation is a well-described phenomenon that paradoxically is a sign of active functional transcription involving the receptors [5].

The influence of concomitant or recent pregnancy on breast cancer prognosis is complex. On the whole, women diagnosed and definitively treated for their breast cancer during pregnancy have no significant differences in survival after adjusting for age and stage at diagnosis, which suggests that pregnancy is not an independent risk factor per se $[1,2]$. On the other hand, breast cancers diagnosed during pregnancy tend to have a higher risk for nodal involvement, higher grade, and more advanced stage at diagnosis [2]. Poor prognosis is often attributed to delayed diagnosis, since masses in the breast may be masked by normal gestational changes. However, an underlying alteration of the tumor biology contributing to poor prognosis of breast cancers diagnosed during or in recent proximity to pregnancy may reflect the incompletely understood influences of hormones, which increase several fold during the course of pregnancy [6]. More recently, a distinction between the prognoses of PABC diagnosed during pregnancy compared to cases diagnosed up to 5 years postpartum has been reported, with postpartum diagnoses having a poorer prognosis not attributable to standard clinical prognostic factors [1]. There is a significant body of evidence supporting the existence of a delay in diagnosis of breast cancer in pregnancy which would result in a transfer of some pregnant women with breast cancer into the postpartum group. Therefore, more studies need to be done to distinguish tumor growthmediated effects occurring during pregnancy and following pregnancy.

To define possible roles of gestational hormones on breast cancer, we evaluated gene expression patterns in PABC from patients diagnosed during pregnancy or $<2$ month postpartum, vs. age-matched, non-PABC controls, defined as at least 5 years from last completed childbirth. The two major cell populations of the breast are epithelial, which line the ducts and milk-producing lobules and are considered to be the major targets of pregnancy hormones, and stromal, which contribute connective tissue elements including vasculature, immune cells, basement membrane, and extracellular matrix (ECM). To differentiate between malignant epithelium and tumorassociated stroma, the two cell types were separated by laser-capture microdissection (LCM) in tumors and adjacent normal tissues. We find that compared to non-PABC, the epithelia of PABC have enhanced expression of genes, many hormone regulated, associated with immune responses and cell cycle regulation. Malignant stroma of PABC is 
characterized by hormone mediated processes related to immune response, cell signaling and factors associated with cell proliferation, and cell death.

\section{Methods}

Tissue Acquisition Surgical specimens were obtained through application to and approval from the University of Colorado Cancer Center Tissue Bank. The tissue bank operates under a prospective, institutional review boardapproved protocol that includes patient consent for the use of normal breast and tumor tissue for research purposes. These cases were provided with full clinical annotation for the pertinent characteristics of the patient and their tumor diagnosis. Cases were identified from the tissue bank as under age 45, diagnosed with an invasive breast cancer, and having fresh frozen tissue available prior to any systemic therapy. Cases were stratified into PABC or non-PABC based on their obstetrical history. PABC was defined as diagnosed during pregnancy while nonPABC were cases in either nulliparous women or women beyond 5 years postpartum at diagnosis. The definition was liberalized for adjacent normal epithelium, with cases added that were less than 2 years postpartum and controls that were more than 2 years postpartum. Tumors and adjacent normal tissues had been flash frozen in liquid nitrogen and stored at $-8{ }^{\circ} \mathrm{C}$.

Laser Capture Microdissection Frozen tissues were embedded in OCT medium (Sakura Finetek, Torrance, CA) and cut at $8 \mu \mathrm{m}$ in a $-2{ }^{\circ} \mathrm{C}$ cryostat. Sections were adhered to Fisher brand Superfrost charged slides (Fischer Scientific, Hampton, NH, USA), immediately placed on a flat piece of dry ice, and stored in a chilled slide box at $-8{ }^{\circ} \mathrm{C}$. A reference slide was created for each specimen. Sections were stained with hematoxylin and eosin and analyzed by a pathologist who marked the exact location in the epithelium and stroma to be dissected. Unstained serial sections underwent dehydration in a graded series of ethanol followed by xylene using a HistoGene LCM Frozen Section Staining Kit (Arcturus) according to the manufacturer's instruction (staining step was not performed). RNAse inhibitor was included in all dehydration solutions except xylene, in which it is insoluble. Slides were air dried for $5 \mathrm{~min}$ and subjected to LCM. Epithelial and stromal cells were separated by microdissection (Pixcell II LCM system, Arcturus, Mountain View, CA, USA) within $1 \mathrm{~h}$ of tissue dehydration. Cells were marked and captured at $10 \times$ microscopy using a 13-16 $\mu \mathrm{m}$ diameter laser. At least 2,000 cells were collected into CapSure HS LCM caps and extracted to RNase-free microtubes using extraction buffer.
Gene Expression Profiling RNA was isolated using the PicoPure RNA isolation Kit (Arcturus) and RNA 28S/18S rRNA peak quality was assessed (Bioanalyzer 2100, Agilent Technologies). RNA was amplified with the WT-Ovation FFPE System based on Ribo-SPIA technology (Nugen Technologies, Inc.), which enables mRNA amplification from as little as $500 \mathrm{pg}$ of total RNA. Single-stranded cDNA were quantified using NanoDrop ND-100 (Nugen Technologies, Inc.). Target preparation for microarray analysis used $5 \mu \mathrm{g}$ of amplified cDNA, which was then fragmented and labeled using FL-Ovation cDNA Biotin Module V2 (Nugen). Fragmented and biotin-labeled targets were hybridized to HG-U133 Plus 2.0 (Affymetrix) gene chips for analysis of over 54,000 probe sets. Microarray data have been submitted to Gene Expression Omnibus (accession number GSE31192).

Microarray Data Normalization Probe sets level data were preprocessed and normalized using robust multi-array average [7], available in the BioConductor package. Probe sets representing the same gene were collapsed based on maximum values.

Hierarchical Clustering To perform hierarchical clustering, we used the CLUSTER 3.0 program and visualized the cluster dendrogram in JavaTree. For statistical significance test of the clusters, we used the $\mathrm{R}$ package pvclust, with 10,000 boostraps. Clusters were considered as statistically significant if approximately unbiased $p$ values of $<0.05$.

Differentially Expressed Genes Analyses The signal-tonoise ratio (SNR) [8] was used to identify differential expressed genes in each comparison. Let $\left[\mu_{1}(g), \mu_{2}(g)\right]$ and $\left[\sigma_{1}(g), \sigma_{2}(g)\right]$ denote the means and standard deviation of the $\log$ base 2 of the expression levels of gene $g$. SNR is based on the null hypothesis that the mean of expression level in group 1 is the same as the one in the group $2\left(\mathrm{H}_{0}\right.$ : $\left.\mu_{1}(g)=\mu_{2}(g), \mathrm{H}_{1}: \mu_{2}(g) \neq \mu_{2}(g)\right)$. For each gene $g$, SNR is obtained by $\mathrm{SNR}_{\mathrm{g}}=\frac{\mu_{1}(\mathrm{~g})-\mu_{2}(\mathrm{~g})}{\sigma_{1}(\mathrm{~g})+\sigma_{2}(\mathrm{~g})}$, where 1 is group 1 and 2 is group $2 . \mathrm{SNR}_{\mathrm{g}}$ reflect differences between the groups relative to the standard deviation within the groups. Large values of $\left|\mathrm{SNR}_{\mathrm{g}}\right|$ indicate a strong correlation between the gene expression and the group difference, while the direction of $S N R_{\mathrm{g}}$ either positive or negative corresponds to $g$ being more highly expressed in groups 1 or 2 . Assuming that the values of $S N R_{\mathrm{g}}$ for all genes have a normal distribution, we converted $S N R_{\mathrm{g}}$ values to $Z$ statistic values and used $p$ value $=0.05$ as the cutoff for determining statistically significant response in either positive or negative direction.

Functional Analyses Over-representation analysis of differentially expressed genes by SNR was performed using the 
Database for Annotation, Visualization and Integrated Discovery (DAVID) bioinformatics resources v.6.7 from the National Institute of Allergy and Infectious Diseases (NIAID/NIH) [9] to identify enriched gene ontology (GO) [10] categories and/or pathways. The predefined pathways we used are compiled from the Kyoto Encyclopedia of Genes and Genomes (KEGG) database (2007 August version) [11]. The KEGG gene sets database contains 395 human pathways that include metabolism, genetic information processing, environmental information processing, cellular processes, and human diseases. The group enrichment score, the geometric mean of member's $p$ values in a corresponding GO group or pathway, is used to rank their functional significance. The GO enrichment analysis was confirmed by Gene Set Enrichment Analysis (GSEA; data not shown) against experimentally validated published genes producing the same functions and pathways from the NIH DAVID [9] with the differentially expressed genes. GSEA offers an unbiased global search for genes that are coordinately regulated in predefined pathways (in this case, as per the KEGG database) [11] with permutation test.

\section{Results}

We speculated that the hormonal milieu of pregnancy contributes to the advanced stage at diagnosis and aggressiveness of PABC [6]. To investigate the molecular nature of this phenotype, cancers of six women diagnosed during pregnancy, and four nonpregnant, age-matched control patients were separated into "epithelial" and "stromal" components by LCM and expression profiled. Table 1 summarizes the clinicopathologic characteristics and ER, PR, HER2, and BRCA1 status of the tumors. All were invasive ductal carcinomas. The majority of patients had no lymph node involvement and stage III disease, with a mean age at diagnosis of 36 years (range, 30-45). All PABC samples were retrieved during pregnancy, except one that was 8 weeks postpartum and all were obtained prior to treatment. In addition to malignant epithelia and tumor-associated stroma, adjacent "normal" tissues were also captured from two PABC and one non-PABC. Paraffin blocks of four additional patients with breast cancer contained only normal tissues. These were nevertheless laser captured and in some cases separated into stromal and epithelial components. Clinicopathologic characteristics of these adjacent normal samples are shown in Fig. 1. In total, 31 tissue samples were profiled.

Unsupervised Hierarchal Clustering To determine the relatedness among the 31 samples, unsupervised hierarchal clustering was performed (Fig. 1a). Samples clustered into two statistically significant clusters $(p<0.05)$ : the first cluster contains "morphologically normal tissue" and "tumorassociated stroma"; whereas the second cluster contains "tumor epithelium" (Fig. 1b). Their separation demonstrates that LCM resulted in highly enriched populations of normal epithelium, tumor, and stroma. Interestingly, only the "normal" group tended to cluster based on parity, irrespective of stromal vs. epithelial type. This indicates that "normal" stroma and epithelia are more closely related to each other than to the same tissue type isolated from tumors. Thus, malignancy confers a unique gene fingerprint to both epithelia and stroma that distinguishes them from normal tissues. Tumor stroma and epithelia stratified separately, with parity not a significant discriminant, and tumor epithelia but not stroma stratified based on ER status. Next, we sought to identify whether the PABC samples were enriched in certain breast cancer subtypes. We performed unsupervised hierarchical clustering of the PABC samples with published breast cancer samples using the intrinsic subtype signature [12]. We found that PABC samples were clustered with different subtypes (i.e., luminal to basal-like; Fig. 1c) and captures the spectrum of breast cancer. The clusters formed by the hierarchical clustering were statistically significant $(p<0.05)$.

Epithelial-Specific Genes of PABC vs. non-PABC Next, to determine the differentially expressed genes between PABC and non-PABC, we performed candidate genes discovery approach using SNR method. From this analysis, 1,097 genes were identified as differentially expressed between the two tumor types, with 570 genes upregulated and 527 downregulated in PABC at a significance of $p<0.05$. A heat map is shown in Fig. 2a and a complete gene list is in Electronic Supplementary Material (ESM) Table 1. These data demonstrate that the underlying gene expression patterns between PABC and non-PABC are distinct from one another. Functional analysis of over-represented annotations and pathways [9, 13] (ESM Table 2a1 and a2) showed that biological processes overrepresented in PABC include "cellular lipid catabolism", "response to molecules of biological origin", "lymphocyte activation", "positive regulation of the immune system", and "leukocyte differentiation", plus genes related to cytokine activity. Biological processes underrepresented in PABC were "appendage and limb morphogenesis" and "protein metabolism". These data suggest increased expression of immune response mediators in PABC.

Hormone-Regulated Gene Signatures in PABC Epithelium To assess the role of pregnancy-associated steroid hormones, estrogen (E)- and progesterone (P)-regulated genes among the 1,097 pregnancy-associated genes in Fig. 2a were compared to the union of six, previously published independent 
Table 1 Patient characteristics

\begin{tabular}{|c|c|c|c|c|c|c|c|c|c|c|c|}
\hline & Patient & $\begin{array}{l}\text { Pregnancy } \\
\text { history }\end{array}$ & Age & $\begin{array}{l}\text { Invasive Ductal } \\
\text { Carcinoma }\end{array}$ & $\begin{array}{l}\text { Adjacent } \\
\text { Normal }\end{array}$ & $\begin{array}{l}\text { Lymph } \\
\text { Node Status }\end{array}$ & $\begin{array}{l}\text { Tumor } \\
\text { Grade }\end{array}$ & TumorER & TumorPR & TumorHER2 & $\begin{array}{l}\text { Tumor } \\
\text { BRCA1 }\end{array}$ \\
\hline \multirow[t]{7}{*}{ PABC } & 52 & Pregnant & 35 & Epi,Str & - & 0 & 2 & + & + & - & BRCA2 \\
\hline & 20 & Pregnant & 36 & Epi,Str & - & 0 & 3 & - & - & - & BRCA1 \\
\hline & 55 & Pregnant & 31 & Epi,Str & - & 0 & 2 & + & + & - & $\mathrm{Neg}$ \\
\hline & 29 & Pregnant & 30 & Epi,Str & - & 0 & 3 & - & - & - & BRCA2 \\
\hline & 103 & $\begin{array}{l}8 \text { Weeks } \\
\text { post }\end{array}$ & 33 & Epi,Str & Epi,Str & $>10+$ & 3 & - & - & - & $\mathrm{Neg}$ \\
\hline & 72 & Pregnant & 39 & Epi,Str & Epi,Str & 0 & 3 & + & + & + & $\mathrm{Neg}$ \\
\hline & 47 & Pregnant & 42 & - & Epi,Str & 0 & 1 & + & + & - & NT \\
\hline \multirow[t]{7}{*}{ Non-PABC } & 25 & No (6 years) & 37 & Epi,Str & - & 0 & 2 & + & - & - & Neg \\
\hline & 49 & No (14 years) & 44 & Epi,Str & - & 0 & 3 & + & + & - & BRCA 1 \\
\hline & 24 & No (nullip) & 28 & Epi,Str & - & 0 & 3 & - & - & - & BRCA 1 \\
\hline & 57 & No (7 years) & 38 & Epi & - & 0 & 3 & + & + & - & NT \\
\hline & 37 & No & 31 & - & Epi,Str & $1+$ & 3 & - & - & - & NT \\
\hline & 19 & No & 45 & - & Epi,Str & 0 & 3 & - & - & - & NT \\
\hline & 21 & No (11 years) & 43 & - & $\mathrm{i}$ & 0 & 3 & + & + & + & NT \\
\hline
\end{tabular}

Epi epithelial cells, Str stroma cells, NT no known test results, $x$ years years since last completed pregnancy

lists of E- or P-regulated genes, generated by us [14-18] and others $[19,20]$. Of these, the majority $(71 \%)$ matched the current dataset. There were 113 E-regulated (Fig. 2b) and 78 P-regulated genes (Fig. 2c) whose clustering dendrograms are shown in Fig. 2, and whose gene names and fold changes are listed in ESM Table $2 \mathrm{~b}$ and c. E- and P-regulated genes distinguish between tumors from pregnant vs. nonpregnant women suggesting that hormones play a role in the distinct biological behavior of the two types of breast cancers. The most abundant functional process among E-regulated genes was "regulation of cell proliferation" with 13 genes differentially expressed in pregnancy (ESM Table 3a). Among P-regulated genes the top category was "regulation of cellular metabolism" with 22 genes differentially expressed (ESM Table 3b). Importantly, increased cell proliferation and metabolism are hallmarks of tumor aggressiveness and poor outcome [21].

Tumor Progression Takes Different Genomic Pathways in Epithelia of PABC vs. Non-PABC Nine hundred seventynine genes differed between normal and malignant epithelia of non-PABC and 1,075 differed in PABC. Only 200 of these overlapped, however, suggesting that some of these genes are likely to be related to differences in the neoplastic process between PABC vs. non-PABC. A list of up- and downregulated genes in epithelia of both $\mathrm{PABC}$ and nonPABC compared to adjacent normal epithelia as baseline are shown in ESM Tables 4a-d. Based on functional analysis, the biological processes associated with these differentially expressed genes are shown in ESM Table 5a-d. The most significantly up- or downregulated gene ontology terms in malignant epithelia of non-PABC (blue bars) and PABC (red bars) when compared to adjacent normal epithelium are shown in ESM Fig. 1. Upregulated in PABC were genes involved in "M phase", "cell cycle", "nuclear division", and "mitosis". Only subtle differences were observed between the two types of cancers for downregulated genes. However, several tumor suppressor genes (phosphatase and tensin homolog, caveolin 1, growth arrest-specific protein 1, p63 and a large number of ribosomal genes (RPL17, RPL35, RPS8, RPS7, RPL18A, RPL32, RPL6, RPL13A, RPS12, RPL3, RPL10, RPL10A, RPL4)) were under-expressed in PABC compared to adjacent normal epithelium. In some cancers, low expression of ribosomal proteins correlates with a more aggressive clinical course; high expression correlates with a more favorable outcome [22]. Of note, while there are several cancer-related pathways associated with tumor progression for both $\mathrm{PABC}$ and non-PABC, an additional upregulated pathway unique to $\mathrm{PABC}$ involves cell cycle-related genes.

Hormones Regulate Unique Sets of Genes in Adjacent Normal Epithelia vs. Tumors Our studies allowed us to identify E- and P-regulated genes differentially expressed between adjacent normal epithelia, and malignant epithelia of PABC or non-PABC (ESM Table 6a-b) and the functional categories associated with these genes (ESM Table $6 \mathrm{c}-\mathrm{d}$ ). Figure 3a represents E-regulated genes that differed between normal vs. non-PABC epithelia (blue) and normal vs. PABC epithelia (red). E-regulated genes are shown in yellow. Different sets of genes were E-regulated in non-PABC compared to adjacent normal as baseline (115 unique genes) and in PABC (128 unique genes) compared to adjacent normal as baseline, with 26 overlapping. E-regulated genes among 
A

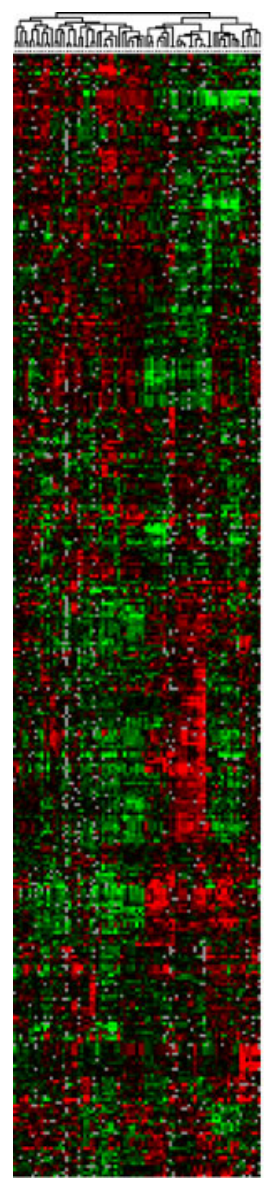

B

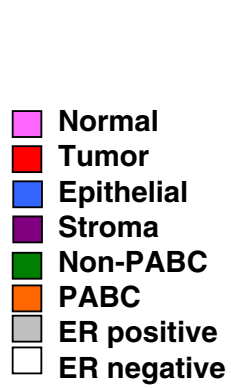

Morphologically Tumor Associated Epithelial Tumor Normal Tissue Stroma

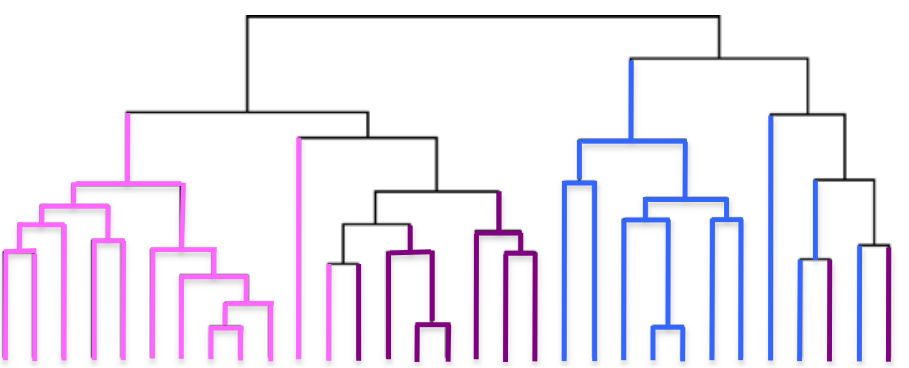

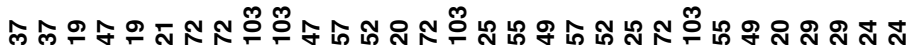

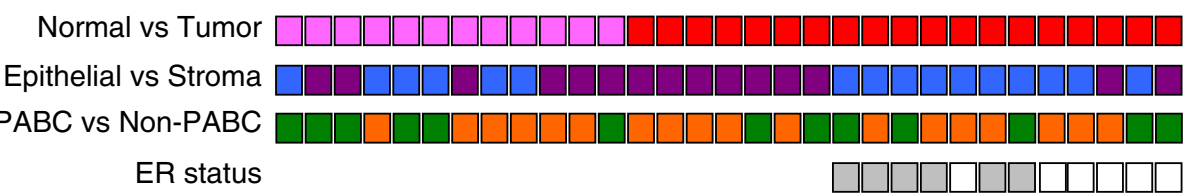

C

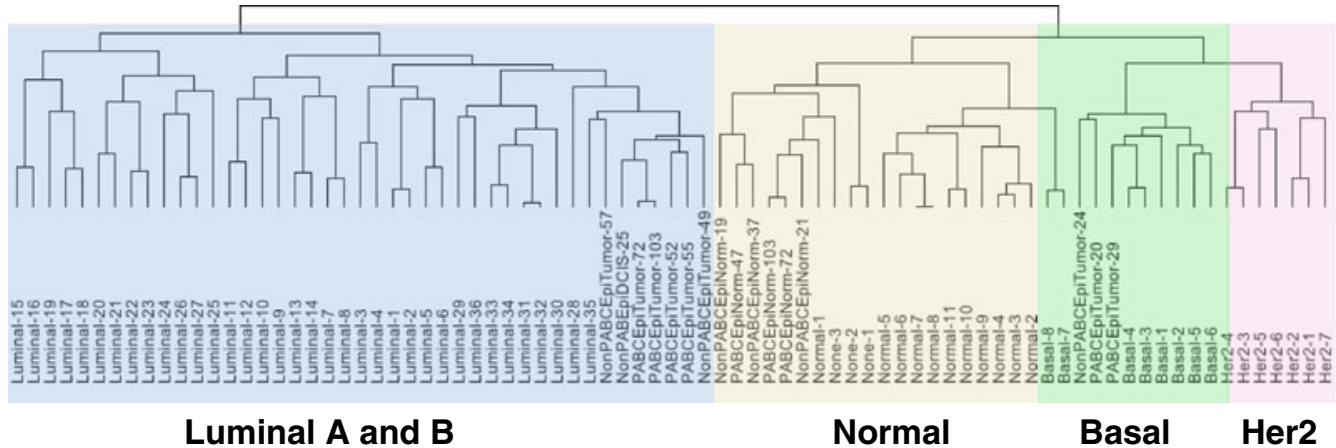

Fig. 1 Unsupervised hierarchical clustering reveals samples cluster primarily on tumor status and cell type. a Unsupervised hierarchical clustering of human breast tumor samples analyzed using Affymetrix HG-U133 Plus 2.0 gene chips as described in the "Methods" section. To avoid redundancy, a single probe set was used for each gene. b Hierarchal clustering of 20,761 genes revealed three main groups: "normal", "tumor associated stroma", and "epithelial tumor". A scaled-down representation of the entire cluster is shown. Branches are color coded according to the group with which the corresponding tumor sample showed the highest correlation. Samples are also color coded based on normal (pink) versus tumor (red), epithelial (blue) versus stroma (purple), PABC (orange) versus non-PABC (green), and ER-positive (gray) versus ER-negative status (white). c Dendogram showing PABC samples cluster with different breast cancer subtypes. Samples are color coded based on luminal A/B (blue), normal (light brown), basal (green), her2 (light pink) the 1,075 differentially expressed PABC genes were dominated ( $50 \%$ ) by mitosis, cell cycle, and $\mathrm{M}$ phase regulation, while fewer than $10 \%$ fell into this category in normal tissues of non-PABC. This suggests that in PABC epitheli$\mathrm{um}$, the increased $\mathrm{E}$ of pregnancy is stimulating massive proliferative changes.

We carried out a similar comparison of P-regulated genes (Fig. 3b) in non-PABC (blue) or PABC (red) epithelia compared to adjacent normal epithelium. There were 51 and 71 unique genes in non-PABC and PABC compared to adjacent normal, respectively, with 23 common to both. The top pathways enriched among P-regulated genes in normal epithelium associated with PABC were also ones regulating cell proliferation. Overall, the data support the conclusion that in addition to targeting tumor cells, $\mathrm{E}$ and $\mathrm{P}$ contribute to tumor aggressiveness by targeting cell cycle and cell proliferation pathways in "normal" epithelia adjacent to PABC.

Stromal Genes in PABC vs. Non-PABC To analyze the microenvironment of $\mathrm{PABC}$ vs. non-PABC, their surrounding tumor-associated stroma were isolated and profiled. Compared to malignant epithelia in which 1,097 genes were differentially expressed between the two tumor types, 331 genes were significantly $(p<0.05)$ different between stroma of the two tumor types with 195 uniquely upregulated and 136 downregulated in PABC-associated stroma. A heat map (Fig. 4a) demonstrates the ability of the 331 genes to discriminate between stroma of PABC and non-PABC. These genes (ESM Table 7) consist of many known stromal-related genes including chemokine CXCL13 [23], chemokine ligand CCL20 [23], metalloproteinases MT3-MMP/MMP16 


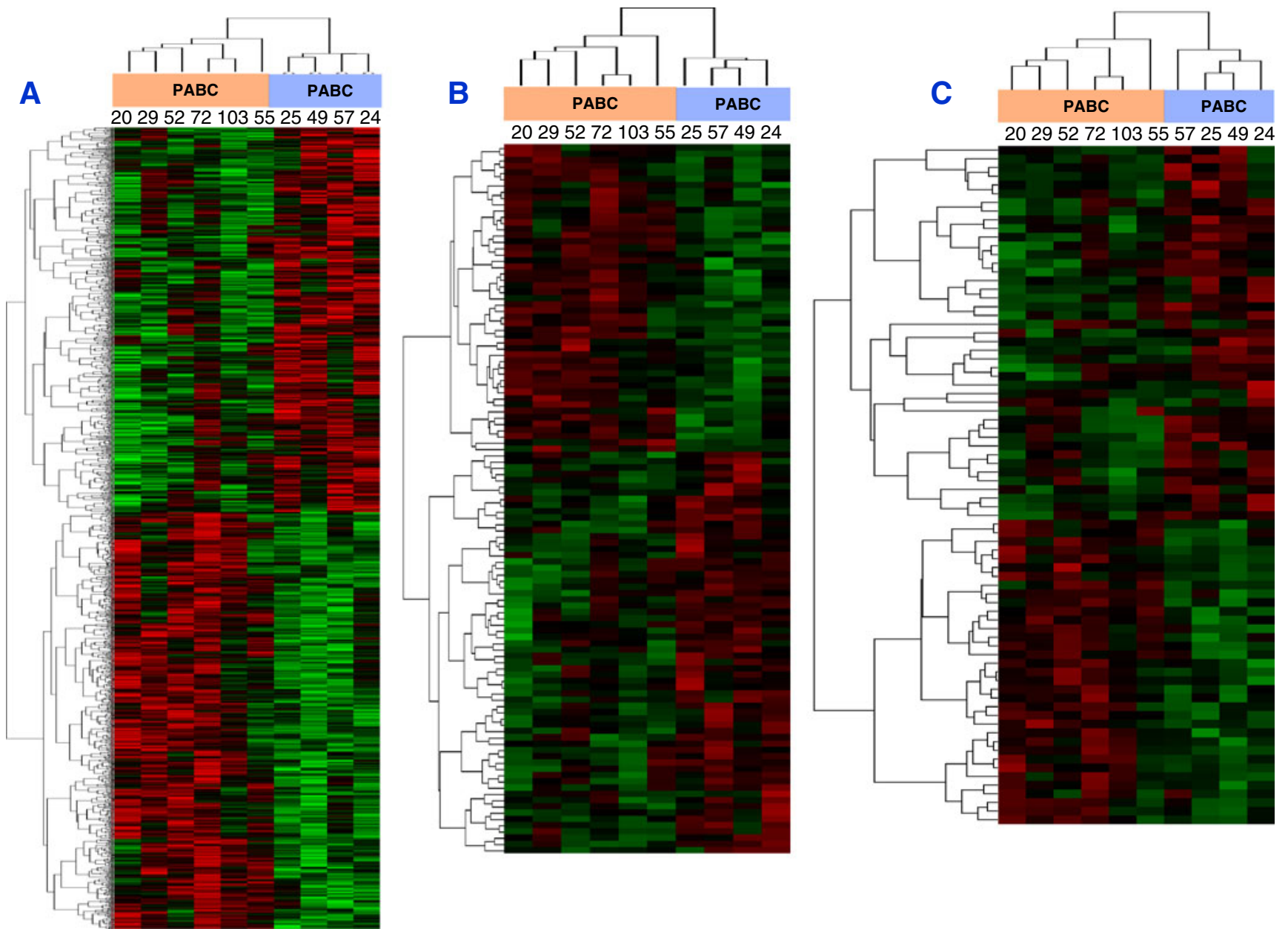

Fig. 2 Subsets of E- and P-regulated genes clustered samples based on pregnancy status in epithelial tumors. Each column represents a single sample and each row a single gene. Red upregulated expression, green downregulated expression. a Hierarchal clustering of the most highly up- or downregulated differentially expressed genes $(1,097)$ identified

[24], ADAMTS15 [24], and several components of the ECM: collagens including COL2A1 [24], COL6A1 [24] and COL9A2 [24], integrin associated protein CD47 [24], and the elastin EMILIN2 [24]. Interestingly, both COL6A1 [25] and ADAMTS15 [26] predict survival in human breast cancers, and according to Nagahara et al. [27] CD47 predicts breast cancer recurrence.

Major biological processes $[9,13]$ (ESM Table 8a1) overrepresented in tumor-associated stroma of PABC compared to non-PABC were "immune system development", "phospholipid catabolic process", "hematopoietic or lymphoid organ development", "lymphocyte activation", "leukocyte differentiation", and "B cell activation". Enrichment of immune response genes was also observed in PABC epithelium (ESM Table 2a) and is consistent with an influx of immune cells during breast remodeling associated with pregnancy, which may contribute to breast cancer progression [28]. Top biological processes associated with genes between PABC and non-PABC epithelium. b Hierarchal clustering of 113 E-regulated gene subset and $\mathbf{c} 78$ P-regulated gene subset differentially expressed between PABC and non-PABC. E-and P-regulated genes were identified as described in the "Methods" section

downregulated in stroma of PABC compared to non-PABC (ESM Table 8a2) were "angiogenesis", "vascular development", "regulation of cellular carbohydrate metabolic process", and "blood vessel morphogenesis" suggesting a paradoxical suppression of angiogenic signaling by tumor-associated stroma in PABC as reported previously by Ma et al. [29].

Hormone-Regulated Gene Signatures in PABC-Associated Stroma Recent data show that low levels of ER are found in breast tumor-associated stroma [29]. We identified 25 Eregulated (Fig. 4b) and 20 P-regulated (Fig. 4c) genes differentially regulated in stroma associated with $\mathrm{PABC}$ vs. non-PABC. Both E- and P-regulated datasets separated $\mathrm{PABC}$ - and non-PABC-associated stroma into distinct clusters (Fig. $4 b$ and $c$ ) and their gene names and fold changes are listed in ESM Tables $8 \mathrm{~b}-\mathrm{c}$. Interestingly, ER $\alpha$ transcripts were present in all stromal samples and did not differ 

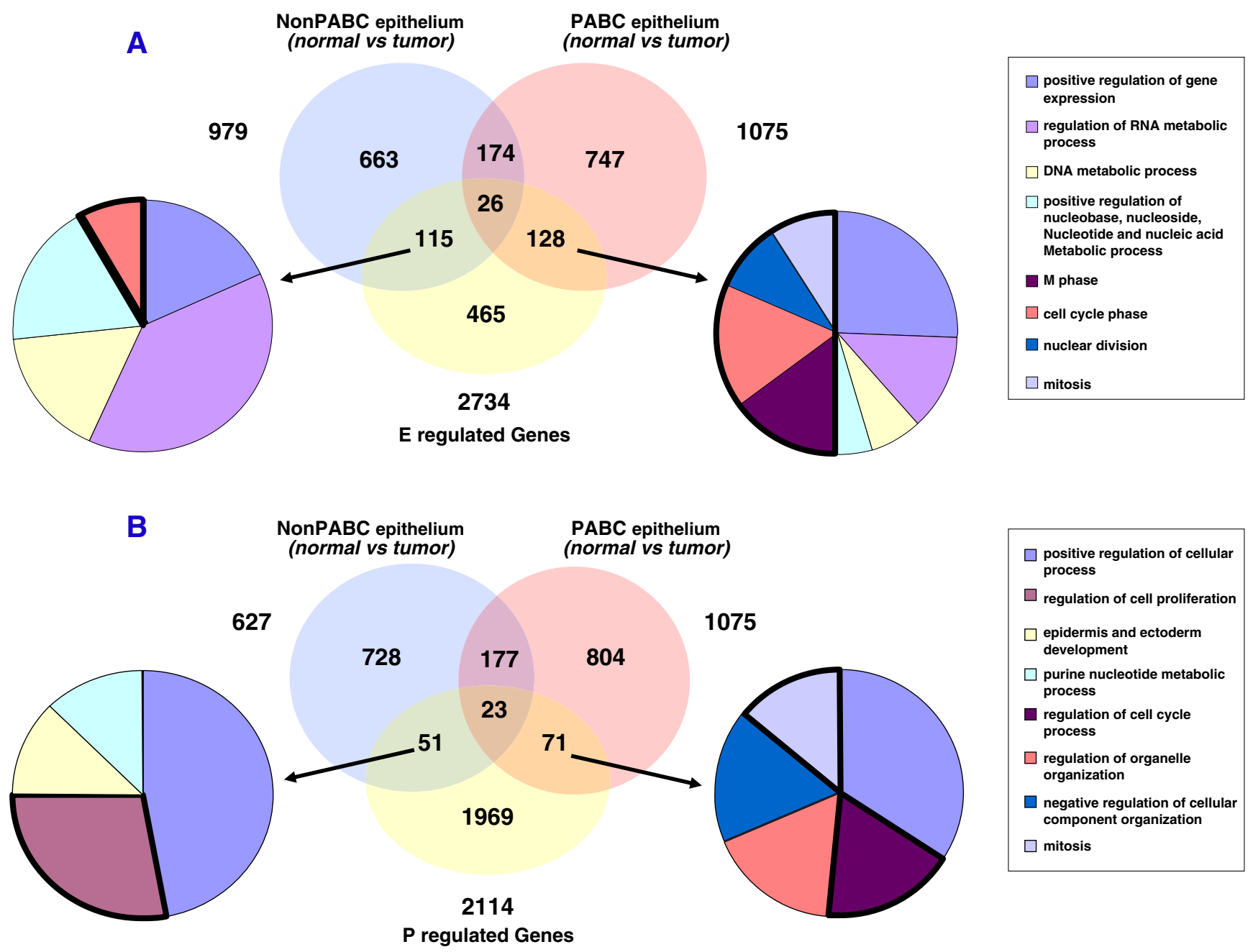

1075

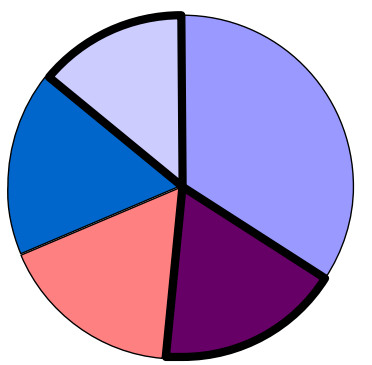

\begin{tabular}{|l}
$\square$ positive regulation of cellular \\
process \\
$\square$ regulation of cell proliferation \\
$\square$ epidermis and ectoderm \\
development \\
$\square$ purine nucleotide metabolic \\
process \\
$\square$ regulation of cell cycle \\
process \\
$\square$ regulation of organelle \\
organization \\
$\square$ negative regulation of cellular \\
component organization \\
$\square$ mitosis
\end{tabular}
Fig. 3 Venn diagram shows little overlap of hormone-regulated genes associated with tumor progression in the epithelium of PABC compared to non-PABC tumors. Venn diagrams display the number of genes associated with tumor progression in $\mathrm{PABC}$ (red) and non-PABC (blue) epithelium. The intersection indicates

significantly between PABC and non-PABC (data not shown). Molecular biological processes associated with these E- and P-regulated genes are listed in ESM Table 9a-b. Among these stromal genes are several encoding chromatinassociated proteins and proliferative factors. Many are also associated with cell signaling pathways mediated by integrins, neuropeptides, p53, wnt, chemokines and cytokines, JAK-STAT, and TGF $\beta$.

Tumor Progression Involves Different Genomic Pathways in $P A B C$ vs. Non-PABC-Associated Stroma Compared to Normal We studied gene expression differences in tumorassociated stroma of PABC (red) and non-PABC (blue) compared to genes in stroma of histologically normal inter- and intralobular regions of the breast A total of 1,217 genes were differentially regulated in normal vs. PABC-associated stroma; 1,119 differed in normal vs. non- the number of probe sets that were changed by both experimental groups for a E-regulated genes and b P-regulated genes. Pie charts indicate the top functional categories represented among the hormone-regulated genes identified in each group. The bold lines indicate related functional categories

PABC-associated stroma. Comparisons between "normal" stroma derived from either PABC or non-PABC yielded 945 genes that were different (ESM Table 10a-c). We found very little (144 combined genes) overlap among "normal" stromal genes vs. tumor-associated stromal genes of either PABC or non-PABC (113 genes), or even between the two types of "normal" stroma (31 genes). This finding is analogous to our finding in "normal" epithelia.

Biological processes up- or downregulated in tumorassociated stroma of non-PABC (blue bars) and PABC (red bars) are shown in ESM Fig. 2 (see also ESM Table 11a-d). The top gene ontology categories among upregulated genes were "positive regulation of immune response", "inflammatory response", and "immune response-regulating signal transduction". Indeed, among 112 "macrophage response" genes recently reported [30] to be consistently seen in a subset of breast cancers, 62 were present in our dataset. 


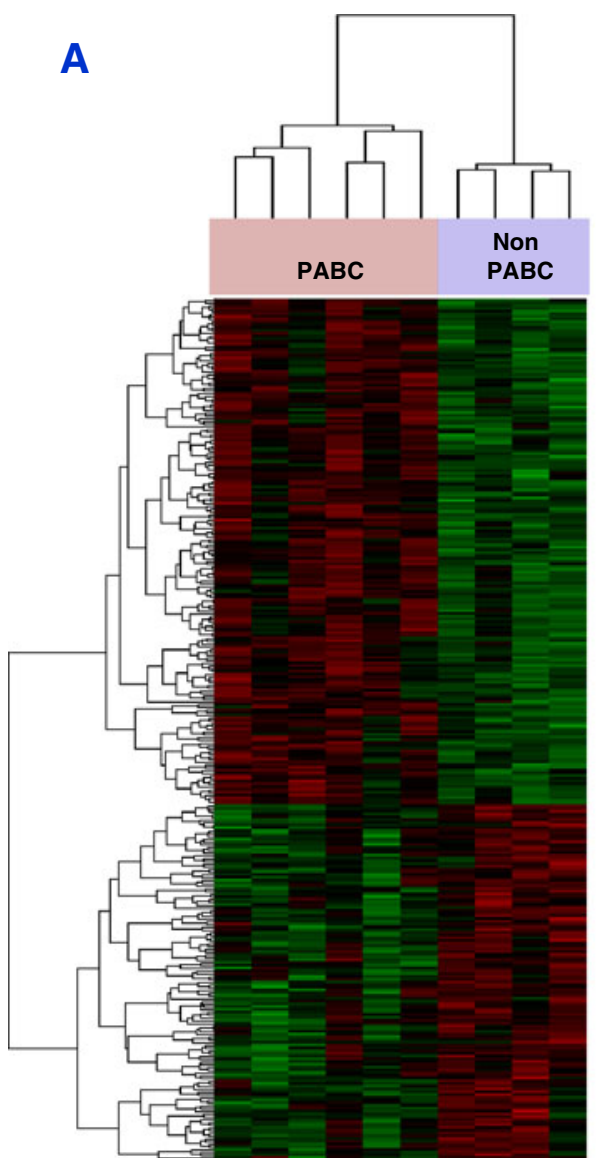

Fig. 4 Subsets of E- and P-regulated genes clustered samples based on pregnancy status in tumor associated stroma. Each column represents a single sample and each row a single gene. Red upregulated expression, green downregulated expression. a Hierarchal clustering of the most highly up- or downregulated differentially expressed genes $(1,097)$

Downregulated genes in tumor-associated stroma (ESM Fig. 2) tended to be associated with "vasculature development", "blood vessel morphogenesis", "response to hormone stimulus", and "chordate embryonic development". Many were unique to PABC (red bars). Interestingly, several HOX genes (HOXD9, HOXA3, HOXA4, HOXD3, HOXD4, HOXA7, HOXD10, and HOXA9) were underexpressed in PABC-associated stroma compared to non-PABC. Similar losses have been correlated with tumor development and progression [31].

Also of note, genes involved in "extracellular matrix" (28), "cell-cell adhesion" (20), and "tight junctions" (12) were more commonly downregulated in $\mathrm{PABC}$, suggesting that its heightened aggressiveness may involve ECM degradation and predisposition to metastasis. Indeed, genes encoding ECM and ECM-modifying proteins correlate with poor breast cancer prognosis [24], 20\% of which are upor downregulated in our datasets. Our dataset also contain $24 \%$ of genes in a "stromal gene signature" related to tumor progression [29]. In general, these genes are two to three times more often identified in stroma of PABC than in identified between PABC and non-PABC. b Hierarchal clustering of 25 E-regulated gene subset and c 20 P-regulated gene subset differentially expressed between PABC and non-PABC. E-and P-regulated genes were identified as described in the "Methods" section

non-PABC. Together, these data point to a decrease in ECM proteins and an increase in immune activity and macrophage response in $\mathrm{PABC}$-associated stroma.

Hormones Regulate Unique Sets of Genes in PABC Stroma Venn diagrams quantify E- (Fig. 5a) and P-regulated (Fig. 5b) genes differentially expressed between "normal" stroma, and PABC-, or non-PABC-associated stroma. As observed in the epithelium, hormones regulate unique sets of genes (ESM Table 12a and b) in normal vs. tumor-associated stroma. E-regulated terms that dominate in PABC-associated stroma include "response to nutrient levels", "positive regulation of myeloid cell differentiation", "negative regulation of cellular metabolic process", "negative regulation of nucleoside, nucleotide and nucleic acid metabolic process", and "regulation of cell death". Important gene sets were also associated with "regulation of cell communication", "toll-like receptor signaling", and "chemokine signaling".

P-regulated processes enriched in stroma of PABC compared to "normal" stroma tended to be "negative" as for 
A
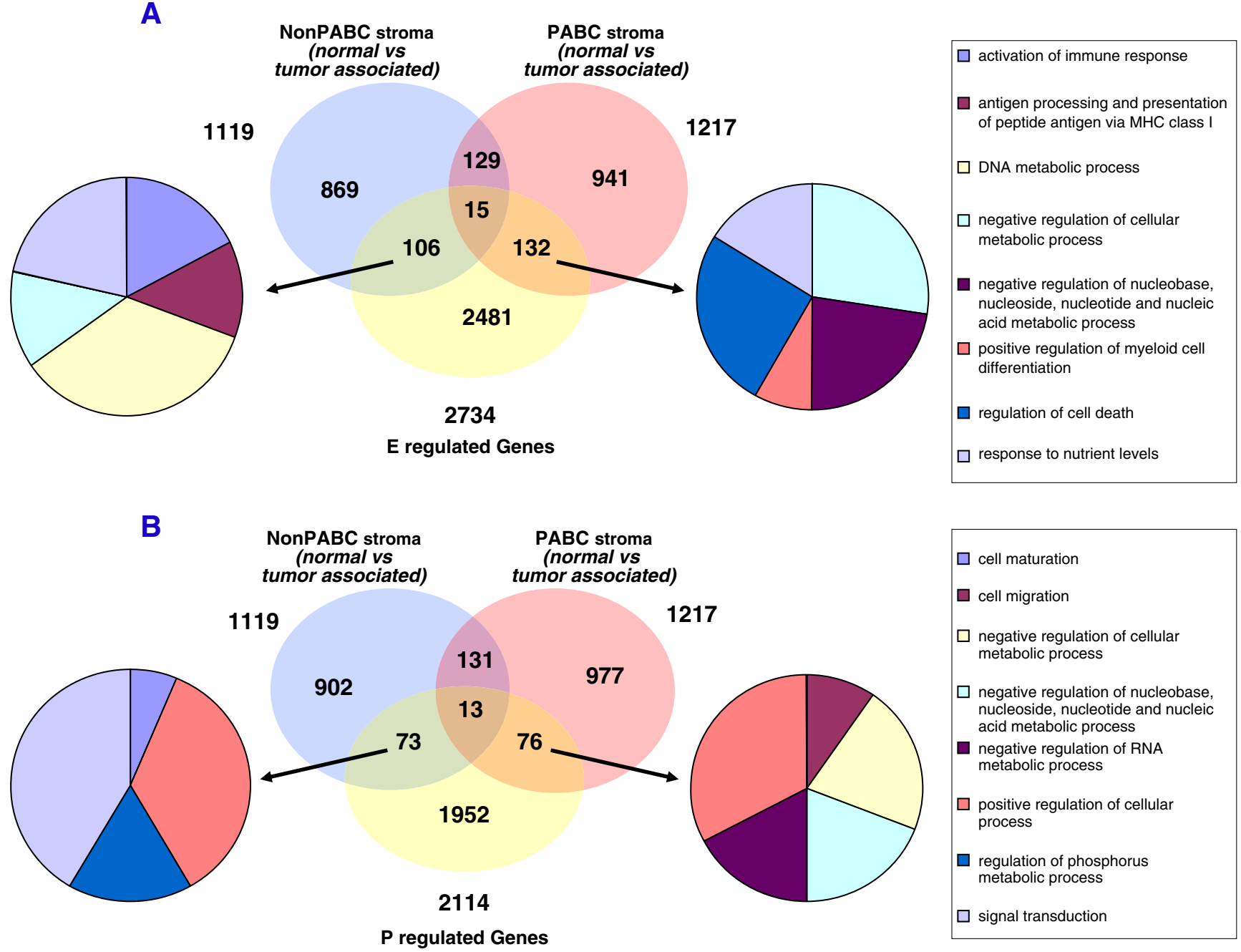

Fig. 5 Venn diagram shows little overlap of hormone regulated genes associated with tumor progression in the stroma of PABC compared to non-PABC tumors. a Venn diagrams display the number of genes associated with tumor progression in the stroma of PABC (red) and non-PABC (blue). The intersection indicates

example "negative regulation of RNA metabolic process", "negative regulation of gene expression", "negative regulation of nucleoside, nucleotide and nuclei acid metabolism", "negative regulation of nitrogen compound metabolic process", and "negative regulation of macromolecule biosynthetic process". Also among P-regulated genes were some indicative of poor prognosis including: tumor suppressor cyclin-dependent kinase inhibitor 1C (CDKN1C/ p57KIP2), Wilms' tumor suppressor gene (WT1), ataxia telangiectasia mutated (ATM), cyclin E2 (CCNE2), methyl-CpG binding domain protein 2 (MBD2), which plays a critical role in metastasis; and c-abl oncogene 1 , nonreceptor tyrosine kinase 1 associated with tumor invasiveness. The biological processes intrinsic to E- and P-regulated genes differentially expressed in normal vs. tumor-associated stroma in both breast cancer types are listed in ESM Table 12c-f. These data suggest that hormone- the number of probe sets that were changed by both experimental groups for: a E-regulated genes and b P-regulated genes. Pie charts indicate the top functional categories represented among the hormone regulated genes identified in each group

mediated stromal effects on cell death and immune mediators may contribute to tumor growth and progression in PABC.

\section{Discussion}

The present studies used gene expression profiling to test the hypothesis that the noncycling, high steroid hormone levels of pregnancy play a role in breast cancers diagnosed in close association with pregnancy and are responsible for their aggressiveness compared to cancers in matched nonpregnant women. Furthermore, we speculated that not only the malignant epithelium, but also the stromal microenvironment surrounding the tumor, may be involved in the heightened aggressiveness. To test these ideas, tumors from patients with $\mathrm{PABC}$ and matched non-PABC controls 
(Table 1) were microdissected to separate tumor-associated stroma from epithelium, and the two tissues were profiled separately. We find that both epithelia and stroma of PABC are enriched for immune-related and cell cycle-associated genes, many of which are hormone regulated. And, given the differences in the hormonal environment of the pregnant vs. nonpregnant breast, most E- and P-regulated genes differed in the two types of cancers. Additionally, we find little overlap among genes expressed in normal tissues vs. their tumor-associated counterparts in PABC compared to nonPABC. Finally, we find that PABC represent all major breast cancer subtypes, luminal to basal-like, as previously reported [32]. These observations warrant further followup study in a larger cohort to validate these findings.

A limited number of studies have profiled genes in the pregnant breast. Tonetti et al. [33] found altered expression of immune/inflammation-associated genes and speculated that the normal parous breast undergoes a weak but persistent inflammatory process. They suggested that this would contribute to tumor aggressiveness if a cancer were present. Russo et al. [34] defined a pregnancy-associated gene signature that differed from that of parous women with cancer, and nulliparous women with or without cancer, and suggest that this signature can be used to define a woman's lifetime breast cancer risk [34]. Hsiao et al. analyzed PABC and matched non-PABC by immunohistochemistry and comparative genomic hybridization [35]. They observed decreased expression of tumor suppressors in PABC associated with loss of DNA copy number, coupled to a gain in DNA copy number of genes encoding morphogenesis, angiogenesis, and metastasis. We also observe downregulation of tumor suppressors in cancers diagnosed during pregnancy.

\section{Tumor Epithelium}

In breast cancer, five molecular subtypes (luminal A and B, basal-like, HER2 overexpressing, and normal breast-like) have been repeatedly described [36]. We investigated whether these five subtypes were also present in PABC. Despite differences in patient samples and microarray platforms, our results confirmed that these five subtypes are represented in PABC. Our results also show that PABC forms a subclass within the luminal $\mathrm{ER}^{+}$subtype (Fig. 1c). This subclass has never been described and may contribute to differences in clinical outcome. Because these analyses did not take into account experimental batch effects that may occur if a subset of experiments are processed differently, additional experiments on a larger series of cases are necessary to validate these findings.

We speculated that the poor prognosis of PABC is linked to the hormonal milieu of pregnancy and defined E- and Pregulated gene signatures in malignant epithelia of PABC that effectively distinguish them from malignant epithelia of
non-PABC (Fig. 2b). The most abundant functional processes among these hormone-regulated genes were "cell proliferation" and "cell metabolism"; hallmarks of tumor aggressiveness and poor outcome [21]. Multiple hormoneregulated genes differentially expressed in PABC but not involved in proliferation and metabolism, are instead associated with tumor aggressiveness. Furthermore, compared to normal epithelium, in PABC, many hormone target genes that regulate $\mathrm{M}$-phase of the cell cycle and cell proliferation were overexpressed. Among mitosis genes upregulated in malignant epithelia of PABC, four-MKI6, AURKA, BIRC5, and MMP11 - are included in the 16 Oncotype DX genes used to predict breast cancer recurrence [37]. Eleven E target genes, most involved in mitosis and differentially expressed in PABC, are part of a 70 gene breast cancer "Outcome Signature" [38], of which five (RRM2, PRC1, MIK67, KIF2C, and AURKA) are upregulated in hormone-resistant breast cancers [16] and included in a poor outcome prognostic set [21,39]. Increased expression of cell cycle genes is indicative of cell proliferation, rapid growth, and increased risk of metastasis which is known to be associated with poor outcome. Indeed, mitotic activity is an independent prognostic factor in breast cancers that may be more important than other clinical biomarkers as indicators of risk [40].

In addition, several important tumor suppressor genes were downregulated in PABC. Interestingly, tumor protein p63 was downregulated in epithelium of PABC in our data and this has also been reported by others [35]. TP63 is a transcription factor and a member of the p53 family and according to Hsiao et al., its expression is associated with increased invasiveness and aggressiveness of PABC.

Among the top biological processes also enriched in malignant epithelia of PABC compared to non-PABC were lipid metabolism and immune response. During pregnancy, the breast undergoes significant remodeling including loss of lipid droplets within adipocytes that not only reflect changing epithelial demands but may also contribute to breast cancer development or growth. Moreover, a growing body of evidence suggests that modulators of immune responses play a role in cancer progression [41] and these immune mediators may contribute to tumor growth and progression in $\mathrm{PABC}$.

\section{Tumor-Associated Stroma}

Immune Response Like the malignant epithelia of PABC, immune-related genes are overexpressed in tumorassociated stroma of PABC. This is consistent with the expression profiling data of Ma et al. [29] showing that in premenopausal women, stroma of high-grade breast cancers exhibit upregulated immune response genes. Of the genes reported by $\mathrm{Ma}$ et al., 102 (16\%) were enriched in PABC 
compared to normal stroma in our studies; two-fold higher than in stroma of non-PABC. In addition, we find that among the macrophage response genes reported by Beck et al. [30], 62 were enriched in tumor-associated stroma of PABC. Heightened density of tumor-associated macrophages correlates with poor prognosis including increased lymph node involvement and decreased survival [42]. Comparison of our gene list with a "marcophage response signature" demonstrates enrichment of these genes in tumorassociated stroma of PABC; a finding previously reported in mammary glands of pregnant rats [43] and proposed to contribute to poorer prognosis of PABC [44].

Angiogenesis Angiogenesis genes were downregulated in tumor-associated stroma of PABC. This was surprising since angiogenesis is generally associated with heightened cancer invasiveness, but confirms reports by Ma et al. [29], and other data that angiogenic and lymphangiogenic factors are reduced in breast cancers $[29,45]$. It has been proposed that dependence on neo-angiogenesis is limited to early phases of tumor growth, while established tumors recruit preexisting host blood vessels, bypassing angiogenesis [46]

Hormones Although ER and PR are not thought to be expressed in stroma, both E- and P-regulated genes were nonetheless demonstrable in our studies (Fig. 5). In support of this, Ma et al. [29] showed low but detectable levels of $\mathrm{ER} \alpha$ in stromal fibroblasts. Indeed $\sim 10 \%$ of our E-targeted genes (Fig. 5) overlap with a published [29] breast cancer stromal gene dataset. We find that many E- and P-regulated genes differentially expressed in tumor-associated stroma of $\mathrm{PABC}$ are involved in cell signaling. Given the importance of stromal signaling on tumorigenesis, our findings suggest a role for hormones in regulating stromal/epithelial interactions that warrant further study.

Our comparison of adjacent normal stroma to PABCassociated stroma revealed decreased expression of ECMencoding genes in tumor-associated stroma. Degradation of the ECM is a common feature of cancer invasion and metastasis [47] and ECM protein expression correlates with clinical outcome in breast cancer [33]. In addition, during pregnancy, ECM breakdown and remodeling is regulated by hormones, coincident with the expanding epithelium [28]. Our findings are generally consistent with these and suggest a role for ECM alterations in PABC aggressiveness.

Hormones also regulate a unique subset of genes in stroma of PABC and non-PABC, compared to normal stroma. Among E-upregulated tumor-associated stromal genes were ones involved in chemokine signaling which activate MMPs required for ECM degradation, and toll-like receptors that promote cancer progression [48]. Other hormoneregulated chemokine receptors and ligands correlate with breast tumor progression, metastasis and poor prognosis [49]. Thirty-three percent of the E-regulated genes overlap with ones that fall into the category of "positive regulation of cell death" from the adjacent normal vs. PABC upregulated gene lists (Fig. 5). It is possible that hormones mediate autophagy of tumor stroma. This could result in recycled nutrients that fuel cancer cells thereby promoting tumor growth and aggressiveness.

\section{Summary}

Our studies are the first to profile malignant epithelia and tumor-associated stroma of PABC compared to non-PABC. We find molecular distinctions between $\mathrm{PABC}$ and nonPABC that suggest mechanisms for PABC aggressiveness. Additionally, we use datasets from clinical and experimental tumor models to assess the role of pregnancy-associated steroid hormones on gene expression of PABC. These analyses demonstrate that hormone-mediated processes related to cell cycle control in malignant epithelia, and to immune response, ECM degradation and cell death in stroma, are enriched in PABC. Our data provide the first experimental support of a theory that the continuous, high hormone levels of pregnancy may play a role in tumor aggressiveness and advanced-stage disease of PABC; a theory that was evoked to explain the poor prognosis associated with these breast cancers. Despite the fact that our PABC sample number is limited, our ability to assign these tumors to known breast cancer subtypes gives us some confidence in the results. Our findings, if validated by future studies, identify significant differences in genomic pathways important in $\mathrm{PABC}$ and its prognosis that may lead to identification of unique therapeutic targets for this disease.

AcknowledgmentsWe thank Wendy Dye for help with data analysis and acknowledge the use of the Laser Capture Microdissection Core, Microarray Core and Computational Bioscience Program of the University of Colorado Cancer Center. This work was supported by the Avon Foundation for Women, the Breast Cancer Research Foundation, the National Foundation for Cancer Research, and NIH R01 CA02689 (to $\mathrm{KBH}$ ) and by a Building Interdisciplinary Research Careers in Women's Health (BIRCWH) K12 Award (to DMEH).

\section{References}

1. Lyons TR, Schedin PJ, Borges VF (2009) Pregnancy and breast cancer: when they collide. J Mammary Gland Biol Neoplasia 14:87-98

2. Vinatier E, Merlot B, Poncelet E, Collinet P, Vinatier D (2009) Breast cancer during pregnancy. Eur J Obstet Gynecol Reprod Biol 147:9-14

3. Petrek JA (1994) Pregnancy safety after breast cancer. Cancer 74:528-531 
4. Nixon AJ, Neuberg D, Hayes DF, Gelman R, Connolly JL, Schnitt S, Abner A, Recht A, Vicini F, Harris JR (1994) Relationship of patient age to pathologic features of the tumor and prognosis for patients with stage I or II breast cancer. J Clin Oncol 12:888-894

5. Horwitz KB, McGuire WL (1978) Nuclear mechanisms of estrogen action. Effects of estradiol and anti-estrogens on estrogen receptors and nuclear receptor processing. J Biol Chem 253:8185-8191

6. Schedin P (2006) Pregnancy-associated breast cancer and metastasis. Nat Rev Cancer 6:281-291

7. Irizarry RA, Bolstad BM, Collin F, Cope LM, Hobbs B, Speed TP (2003) Summaries of Affymetrix Genechip probe level data. Nucleic Acids Res 31:e15

8. Golub TR, Slonim DK, Tamayo P, Huard C, Gaasenbeek M, Mesirov JP, Coller H, Loh ML, Downing JR, Caligiuri MA, Bloomfield CD, Lander ES (1999) Molecular classification of cancer: class discovery and class prediction by gene expression monitoring. Science 286:531-537

9. Huang DW, Sherman BT, Lempicki RA (2009) Systematic and integrative analysis of large gene lists using david bioinformatics resources. Nat Protoc 4:44-57

10. Ashburner M, Ball CA, Blake JA, Botstein D, Butler H, Cherry JM, Davis AP, Dolinski K, Dwight SS, Eppig JT, Harris MA, Hill DP, Issel-Tarver L, Kasarskis A, Lewis S, Matese JC, Richardson JE, Ringwald M, Rubin GM, Sherlock G (2000) Gene ontology: tool for the unification of biology. The gene ontology consortium. Nat Genet 25:25-29

11. Kanehisa M, Goto S, Furumichi M, Tanabe M, Hirakawa M (2010) Kegg for representation and analysis of molecular networks involving diseases and drugs. Nucleic Acids Res 38:D355-360

12. Perou CM, Sorlie T, Eisen MB, van de Rijn M, Jeffrey SS, Rees CA, Pollack JR, Ross DT, Johnsen H, Akslen LA, Fluge O, Pergamenschikov A, Williams C, Zhu SX, Lonning PE, Borresen-Dale AL, Brown PO, Botstein D (2000) Molecular portraits of human breast tumours. Nature 406:747-752

13. Huang DW, Sherman BT, Lempicki RA (2009) Bioinformatics enrichment tools: paths toward the comprehensive functional analysis of large gene lists. Nucleic Acids Res 37:1-13

14. Sartorius CA, Harvell DM, Shen T, Horwitz KB (2005) Progestins initiate a luminal to myoepithelial switch in estrogen-dependent human breast tumors without altering growth. Cancer Res 65:9779-9788

15. Richer JK, Jacobsen BM, Manning NG, Abel MG, Wolf DM, Horwitz KB (2002) Differential gene regulation by the two progesterone receptor isoforms in human breast cancer cells. J Biol Chem 277:5209-5218

16. Harvell DM, Richer JK, Singh M, Spoelstra N, Finlayson C, Borges VF, Elias AD, Horwitz KB (2008) Estrogen regulated gene expression in response to neoadjuvant endocrine therapy of breast cancers: Tamoxifen agonist effects dominate in the presence of an aromatase inhibitor. Breast Cancer Res Treat 112:489-501

17. Harvell DM, Richer JK, Allred DC, Sartorius CA, Horwitz KB (2006) Estradiol regulates different genes in human breast tumor xenografts compared with the identical cells in culture. Endocrinology 147:700-713

18. Ghatge RP, Jacobsen BM, Schittone SA, Horwitz KB (2005) The progestational and androgenic properties of medroxyprogesterone acetate: gene regulatory overlap with dihydrotestosterone in breast cancer cells. Breast Cancer Res 7:R1036-1050

19. Frasor J, Danes JM, Komm B, Chang KCN, Lyttle CR, Katzenellenbogen BS (2003) Profiling of estrogen up-and downregulated gene expression in human breast cancer cells: insights into gene networks and pathways underlying estrogenic control of proliferation and cell phenotype. Endocrinology 144:4562-4574

20. Creighton CJ, Cordero KE, Larios JM, Miller RS, Johnson MD, Chinnaiyan AM, Lippman ME, Rae JM (2006) Genes regulated by estrogen in breast tumor cells in vitro are similarly regulated in vivo in tumor xenografts and human breast tumors. Genome Biol 7:R28

21. Dai H, van't Veer L, Lamb J, He YD, Mao M, Fine BM, Bernards R, van de Vijver M, Deutsch P, Sachs A, Stoughton R, Friend S (2005) A cell proliferation signature is a marker of extremely poor outcome in a subpopulation of breast cancer patients. Cancer Res 65:4059-4066

22. Durig J, Nuckel H, Huttmann A, Kruse E, Holter T, Halfmeyer K, Fuhrer A, Rudolph R, Kalhori N, Nusch A, Deaglio S, Malavasi F, Moroy T, Klein-Hitpass L, Duhrsen U (2003) Expression of ribosomal and translation-associated genes is correlated with a favorable clinical course in chronic lymphocytic leukemia. Blood 101:2748-2755

23. Douglas MR, Morrison KE, Salmon M, Buckley CD (2002) Why does inflammation persist: a dominant role for the stromal microenvironment? Expert Rev Mol Med 4:1-18

24. Bergamaschi A, Tagliabue E, Sorlie T, Naume B, Triulzi T, Orlandi R, Russnes HG, Nesland JM, Tammi R, Auvinen P, Kosma VM, Menard S, Borresen-Dale AL (2008) Extracellular matrix signature identifies breast cancer subgroups with different clinical outcome. J Pathol 214:357-367

25. Bertucci F, Nasser V, Granjeaud S, Eisinger F, Adelaide J, Tagett R, Loriod B, Giaconia A, Benziane A, Devilard E, Jacquemier J, Viens P, Nguyen C, Birnbaum D, Houlgatte R (2002) Gene expression profiles of poor-prognosis primary breast cancer correlate with survival. Hum Mol Genet 11:863-872

26. Porter S, Span PN, Sweep FC, Tjan-Heijnen VC, Pennington CJ, Pedersen TX, Johnsen M, Lund LR, Romer J, Edwards DR (2006) Adamts 8 and adamts 15 expression predicts survival in human breast carcinoma. Int J Cancer 118:1241-1247

27. Nagahara M, Mimori K, Kataoka A, Ishii H, Tanaka F, Nakagawa T, Sato T, Ono S, Sugihara K, Mori M (2010) Correlated expression of cd47 and sirpa in bone marrow and in peripheral blood predicts recurrence in breast cancer patients. Clin Cancer Res $16: 4625-4635$

28. McCready J, Arendt LM, Rudnick JA, Kuperwasser C (2010) The contribution of dynamic stromal remodeling during mammary development to breast carcinogenesis. Breast Cancer Res 12:205

29. Ma XJ, Dahiya S, Richardson E, Erlander M, Sgroi DC (2009) Gene expression profiling of the tumor microenvironment during breast cancer progression. Breast Cancer Res 11:R7

30. Beck AH, Espinosa I, Edris B, Li R, Montgomery K, Zhu S, Varma S, Marinelli RJ, van de Rijn M, West RB (2009) The macrophage colony-stimulating factor 1 response signature in breast carcinoma. Clin Cancer Res 15:778-787

31. Makiyama K, Hamada J, Takada M, Murakawa K, Takahashi Y, Tada M, Tamoto E, Shindo G, Matsunaga A, Teramoto K, Komuro K, Kondo S, Katoh H, Koike T, Moriuchi T (2005) Aberrant expression of hox genes in human invasive breast carcinoma. Oncol Rep 13:673-679

32. Millikan RC, Newman B, Tse CK, Moorman PG, Conway K, Dressler LG, Smith LV, Labbok MH, Geradts J, Bensen JT, Jackson S, Nyante S, Livasy C, Carey L, Earp HS, Perou CM (2008) Epidemiology of basal-like breast cancer. Breast Cancer Res Treat 109:123-139

33. Asztalos S, Gann PH, Hayes MK, Nonn L, Beam CA, Dai Y, Wiley EL, Tonetti DA (2010) Gene expression patterns in the human breast after pregnancy. Cancer Prev Res (Phila) 3:301311

34. Russo J, Balogh GA, Russo IH (2008) Full-term pregnancy induces a specific genomic signature in the human breast. Cancer Epidemiol Biomarkers Prev 17:51-66

35. Hsiao YH, Su YA, Tsai HD, Mason JT, Chou MC, Man YG (2010) Increased invasiveness and aggressiveness in breast epithelia with cytoplasmic p63 expression. Int J Biol Sci 6:428-442 
36. Sorlie T, Tibshirani R, Parker J, Hastie T, Marron JS, Nobel A, Deng S, Johnsen H, Pesich R, Geisler S, Demeter J, Perou CM, Lonning PE, Brown PO, Borresen-Dale AL, Botstein D (2003) Repeated observation of breast tumor subtypes in independent gene expression data sets. Proc Natl Acad Sci USA 100:8418-8423

37. Paik S, Shak S, Tang G, Kim C, Baker J, Cronin M, Baehner F, Walker M, Watson D, Park T, Hiller W, Fisher E, Wickerham D, Bryant J, Wolmark N (2004) A multigene assay to predict recurrence of tamoxifen-treated, node-negative breast cancer. N Engl J Med 351:2817-2826

38. Yu J, Cordero KE, Johnson MD, Ghosh D, Rae JM, Chinnaiyan AM, Lippman ME (2008) A transcriptional fingerprint of estrogen in human breast cancer predicts patient survival. Neoplasia 10:79-88

39. Xu L, Tan AC, Winslow RL, Geman D (2008) Merging microarray data from separate breast cancer studies provides a robust prognostic test. BMC Bioinforma 9:125

40. Medri L, Volpi A, Nanni O, Vecci AM, Mangia A, Schittulli F, Padovani F, Giunchi DC, Zito A, Amadori D, Paradiso A, Silvestrini R (2003) Prognostic relevance of mitotic activity in patients with node-negative breast cancer. Mod Pathol 16:1067-1075

41. Prestwich RJ, Errington F, Hatfield P, Merrick AE, Ilett EJ, Selby PJ, Melcher AA (2008) The immune system-is it relevant to cancer development, progression and treatment. Clin Oncol 20:101-112

42. Tsutsui S, Yasuda K, Suzuki K, Tahara K, Higashi H, Era S (2005) Macrophage infiltration and its prognostic implications in breast cancer: the relationship with VEGF expression and microvessel density. Oncology reports 14:425-431

43. Zhao W, Grubbs CJ, Myers RK, Nilsen-Hamilton M (2010) Parity is associated with an expanded macrophage population in the mammary gland. Int J Oncol 37:1195-1202

44. O'Brien J, Lyons T, Monks J, Lucia MS, Wilson RS, Hines L, Man YG, Borges V, Schedin P (2010) Alternatively activated macrophages and collagen remodeling characterize the postpartum involuting mammary gland across species. Am J Pathol 176:12411255

45. Boneberg EM, Legler DF, Hoefer MM, Ohlschlegel C, Steininger H, Fuzesi L, Beer GM, Dupont-Lampert V, Otto F, Senn HJ, Furstenberger G (2009) Angiogenesis and lymphangiogenesis are downregulated in primary breast cancer. Br J Cancer 101:605-614

46. Leenders WP, Kusters B, Verrijp K, Maass C, Wesseling P, Heerschap A, Ruiter D, Ryan A, de Waal R (2004) Antiangiogenic therapy of cerebral melanoma metastases results in sustained tumor progression via vessel co-option. Clin Cancer Res 10:6222-6230

47. Ronnov-Jessen L, Petersen OW, Bissell MJ (1996) Cellular changes involved in conversion of normal to malignant breast: Importance of the stromal reaction. Physiol Rev 76:69-125

48. Sato Y, Goto Y, Narita N, Hoon DS (2009) Cancer cells expressing toll-like receptors and the tumor microenvironment. Cancer Microenviron 2(Suppl 1):205-214

49. Ali S, Lazennec G (2007) Chemokines: novel targets for breast cancer metastasis. Cancer Metastasis Rev 26:401-420 